Copyright (C) 2008 IEEE. Reprinted from IEEE Transactions on Signal Processing, 2007; 55 (1):20-31

This material is posted here with permission of the IEEE. Such permission of the IEEE does not in any way imply IEEE endorsement of any of the University of Adelaide's products or services. Internal or personal use of this material is permitted. However, permission to reprint/republish this material for advertising or promotional purposes or for creating new collective works for resale or redistribution must be obtained from the IEEE by writing to pubs-permissions@ieee.org.

By choosing to view this document, you agree to all provisions of the copyright laws protecting it. 


\title{
GLRT-Based Threshold Detection-Estimation Performance Improvement and Application to Uniform Circular Antenna Arrays
}

\author{
Yuri I. Abramovich, Senior Member, IEEE, Nicholas K. Spencer, and Alexei Y. Gorokhov, Member, IEEE
}

\begin{abstract}
The problem of estimating the number of independent Gaussian sources and their parameters impinging upon an antenna array is addressed for scenarios that are problematic for standard techniques, namely, under "threshold conditions" (where subspace techniques such as MUSIC experience an abrupt and dramatic performance breakdown). We propose an antenna geometry-invariant method that adopts the generalized-likelihood-ratio test (GLRT) methodology, supported by a maximum-likelihoodratio lower-bound analysis that allows erroneous solutions ("outliers") to be found and rectified. Detection-estimation performance in both uniform circular and linear antenna arrays is shown to be significantly improved compared with conventional techniques but limited by the performance-breakdown phenomenon that is intrinsic to all such maximum-likelihood (ML) techniques.
\end{abstract}

Index Terms-Adaptive signal detection, antenna arrays, circular arrays, direction-of-arrival estimation, maximum-likelihood estimation.

\section{INTRODUCTION AND BACKGROUND}

$\mathbf{O}$ NE of the significant advantages of high-resolution techniques such as MUSIC and root-MUSIC [1] for direction-finding applications is their invariance to the geometry of the antenna array. For the number of independent (uncorrelated) Gaussian sources that is less than the number of antenna sensors $(m<M)$ and a sufficiently large sample size $N$ and/or signal-to-noise ratio (SNR), high-resolution techniques yield asymptotically optimal direction-of-arrival (DOA) estimation accuracy, which could be only marginally improved upon by strictly maximum-likelihood (ML) methods that incorporate information on the particular antenna array geometry [2], [3]. However, the main practical problem with subspace-based high-resolution methods is that under "threshold conditions" (with insufficient sample support and/or SNR), they experience an abrupt and dramatic accuracy degradation ("performance

Manuscript received March 19, 2004; revised October 9, 2005. The associate editor coordinating the review of this manuscript and approving it for publication was Dr. Jan C. de Munck. Some results of this paper were presented at the Asilomar Conference, Pacific Grove, CA, November 9-12, 2003.

Y. I. Abramovich is with the Intelligence, Surveillance and Reconnaissance Division (ISRD), Defence Science and Technology Organisation (DSTO), Edinburgh, SA, 5111, Australia (e-mail: Yuri.Abramovich@dsto.defence.gov.au).

N. K. Spencer is with the Adelaide Research \& Innovation Pty, Ltd. (ARI), 200 Labs, Intelligence, Surveillance and Reconnaissance Division (ISRD), Defence Science and Technology Organisation (DSTO), Edinburgh, SA, 5111, Australia (e-mail: Nick.Spencer@adelaide.edu.au).

A. Y. Gorokhov is with the Qualcomm Technology \& Ventures (QT \& V), Qualcomm, Inc., San Diego, CA 92121-1714 USA (e-mail: gorokhov@ qualcomm.com)

Digital Object Identifier 10.1109/TSP.2006.882078 breakdown") due to some severely erroneous DOA estimates ("outliers").

Most approaches that try to improve DOA estimation performance in the threshold region incorporate specific properties of the antenna array geometry. In our recent papers on linear antenna arrays [4], [5], we demonstrated that accurate ML estimation allows us to overcome the MUSIC threshold and, in fact, push the threshold conditions all the way to the region where "ML performance breakdown" occurs, i.e., where DOA sets that contain severely erroneous DOA estimates (that are used to compute the covariance matrix model) yield a likelihood ratio (LR) that exceeds that of the true (exact) covariance matrix. Naturally, since such erroneous estimates are believed to be "more likely" than the true parameter, this type of breakdown is intrinsic and could not be overcome at all within the ML paradigm. Still, this important "gap" between, say, MUSIC performance breakdown threshold conditions and those for the ultimate ML performance breakdown, represents a region in which our techniques [4], [5] (developed for linear arrays) can be successfully employed for detection-estimation. Unfortunately, those techniques rely upon certain Toeplitz properties of the linear arrayrelated covariance matrices, and so are not directly applicable to different array geometries. More specifically, we cannot apply these techniques directly for uniform circular arrays (UCAs) that are often used in direction-finding applications, primarily due to their complete azimuthal coverage and almost invariant directional pattern [6], [7].

A recently introduced generalized rectification approach [8] and a global matched-filter technique [9] each improved UCA performance sufficiently to push the threshold conditions towards more difficult scenarios, beyond the conventional MUSIC threshold. In slightly different ways, both techniques take advantage of some structural properties of UCA-related covariance matrices. Nevertheless, the problem of optimum detectionestimation performance near the "threshold region" for UCAs, and in fact for an arbitrary antenna array geometry, has not yet been addressed.

To achieve this goal, we again exploit the generalized-likelihood-ratio test (GLRT) methodology (that is not specific to any particular array geometry) which essentially provides a normalized metric framework for covariance matching. A crucial ingredient in our method is a test that assesses the "quality" of the match between the sample matrix and the covariance matrix produced by candidate models. This test is based on the LR, which is just a normalized likelihood function (LF). An important (and remarkable) property of the LR is that its probability 
density function (pdf) for the true covariance matrix does not depend on this matrix (i.e., is "scenario-free"). This importance is due to the fact that the ML model, which is searched over the admissible set that includes the true parameters, must generate an LR that equals or exceeds the LR produced by the true covariance matrix. Regardless of any particular ML optimization algorithm, therefore, there is a statistical scenario-free "lower bound" in LR with which all candidate optimized solutions can be compared. For example, the MUSIC-generated covariance matrix model can be checked against the lower bound in order to reliably recognize MUSIC outliers (where the LR is below the lower bound). This approach allows us to recognize ("predict") the onset of MUSIC performance breakdown in an arbitrary geometry antenna array; the problem of how to rectify ("cure") outliers is addressed here.

It is important to emphasize that performance breakdown usually occurs for an insufficient sample size and/or SNR, which is the antithesis of the asymptotic conditions for which most theoretical results on ML and subspace techniques (such as MUSIC) have been derived. For example, instead of the single global ML extremum in a convex vicinity of the true covariance matrix, we often observe multiextremal behavior wherein the global extremum is further from the true solution than some local extremum (see [10], for example). In this regard, any solution that is "as likely as" the true one is statistically no worse than the global ML solution for such "preasymptotic conditions."

Since we cannot use any asymptotic properties, it is important to rely on sufficiently accurate nonasymptotic statistical properties of the LR tests. On the other hand, apart from the scenario-free pdf, very little can be done to analytically explore the breakdown conditions for any particular technique and scenario, hence most of our results on performance improvement are demonstrated by simulations.

This paper is organized as follows. Section II formulates the problem, gives background material, and describes our GLRT-based framework for detection-estimation. Section III discusses MUSIC performance breakdown "prediction and cure" in sensor arrays. In fact, the method developed is applicable to arbitrary antenna array geometries, but results for UCAs are presented in Section IV. We also introduce here the results of a comparative performance analysis for our geometry-free "prediction and cure" technique with the ULA-specific LR optimization technique developed in [4] and [5]. Our summary and conclusions appear in Section V.

\section{PROBLEM Formulation}

Consider an $M$-sensor antenna array with omnidirectional sensors located at Cartesian coordinates $\left\{\rho_{k} \cos \left(\delta_{k}\right), \rho_{k} \sin \left(\delta_{k}\right)\right\}$ $(k=0, \ldots, M-1)$. For simplicity, we assume that the array sensors and the sources are coplanar so that we can remain within the one-dimensional DOA estimation problem, similarly to [9]. The particular array geometry we are considering for the "prediction and cure" of MUSIC performance breakdown is a UCA with $\rho_{k}=\rho$ and $\delta_{k}=2 \pi k / M$, where $\rho$ is the radius of the UCA measured in wavelength units $\lambda$. We also briefly consider a ULA, for which $\rho_{k}=k d$ and $\delta_{k}=0$, where $d$ is the intersensor spacing, again measured in wavelength units.
For a UCA, the array-signal manifold ("steering") vector associated with the azimuthal angle (DOA) $\theta \in[0,2 \pi)$ is

$$
\boldsymbol{s}(\theta)=\left[s(\theta), s\left(\theta-\frac{2 \pi}{M}\right), \ldots, s\left(\theta-\frac{2 \pi(M-1)}{M}\right)\right]^{T}
$$

where

$$
s(\theta) \equiv \exp \left[2 \pi i \frac{\rho}{\lambda} \cos \theta\right]
$$

while for a ULA, it is

$$
\boldsymbol{s}(\theta)=\left[s^{0}(\theta), s^{1}(\theta), \ldots, s^{M-1}(\theta)\right]^{T}
$$

where

$$
s(\theta) \equiv \exp \left[2 \pi i \frac{d}{\lambda} \sin \theta\right]
$$

Assuming $m$ independent (uncorrelated) Gaussian sources in the general case, we may express the vector of observed sensor outputs (the "snapshot") at time $t$ as

$$
\boldsymbol{y}(t)=S(\boldsymbol{\theta}) \boldsymbol{x}(t)+\boldsymbol{\eta}(t) \quad \text { for } \quad t=1, \ldots, N
$$

where $\boldsymbol{x}(t) \in \mathcal{C}^{m \times 1}$ are the Gaussian signal amplitudes with DOAs $\boldsymbol{\theta} \equiv\left[\theta_{1}, \ldots, \theta_{m}\right]^{T}$ and powers $P \equiv$ $\operatorname{diag}\left[p_{1}, \ldots, p_{m}\right]>0$, the array-signal manifold matrix is $S(\boldsymbol{\theta}) \equiv\left[\boldsymbol{s}\left(\theta_{1}\right), \ldots, \boldsymbol{s}\left(\theta_{m}\right)\right] \in \mathcal{C}^{M \times m}$, and $\boldsymbol{\eta}(t) \in \mathcal{C}^{M \times 1}$ is Gaussian white noise of power $p_{0}$

$$
\boldsymbol{x}(t) \sim \mathcal{C N}(m, 0, P), \quad \boldsymbol{\eta}(t) \sim \mathcal{C N}\left(M, 0, p_{0} I_{M}\right)
$$

where $\mathcal{C N}(M, 0, R)$ denotes a complex (circular) Gaussian distribution of dimension $M$ with zero mean and covariance matrix $R$. Therefore, the observed data $\boldsymbol{y}(t)$ is described by the complex Gaussian distribution $\mathcal{C N}(M, 0, R)$, where

$$
R=S(\boldsymbol{\theta}) P S(\boldsymbol{\theta})^{H}+p_{0} I_{M} .
$$

We assume that the snapshots are statistically independent

$$
\mathcal{E}\left\{\boldsymbol{y}\left(t_{1}\right) \boldsymbol{y}^{H}\left(t_{2}\right)\right\}= \begin{cases}R & \text { for } t_{1}=t_{2} \\ 0 & \text { for } t_{1} \neq t_{2}\end{cases}
$$

and so the sufficient statistic for any inference regarding this data is the direct data covariance (DDC) (sample) matrix

$$
\hat{R}=\frac{1}{N} \sum_{t=1}^{N} \boldsymbol{y}(t) \boldsymbol{y}^{H}(t)
$$

where $N \hat{R}$ is described by the complex Wishart distribution $\mathcal{C W}(N, M ; R)[11]$.

Suppose that $\mathbb{R}_{\mu}$ is the set of all possible covariance matrices $R_{\mu}$ that have a structure appropriate to model $\mu$ independent Gaussian sources in noise in any particular antenna array. According to the GLRT methodology (see [1, Sec. 4.7.1], for example), for each possible number of sources $\mu$, we need to find the covariance matrix $R_{\mu} \in \mathbb{R}_{\mu}$ that yields the maximum 
LR for the given $\hat{R}$. The LR is designed to test the hypothesis $H_{0}$ that the covariance matrix derived from the snapshots $\boldsymbol{y}(t)$ $(t=1, \ldots, N)$ is proportional to some matrix $C$, against the contrary hypothesis $H_{1}[1]$

$$
R_{\mu}=\arg \max _{C \in \mathbb{R}_{\mu}} L R(C) .
$$

The "nested" GLRT property, which is crucial for its use in detection, is that

$$
L R\left(R_{\mu}\right) \geq L R\left(R_{\mu^{\prime}}\right)
$$

for every $\mu>\mu^{\prime}$. Obviously, it is incorrect to try to select the "best" model by simply choosing $\max _{\mu} L R\left(R_{\mu}\right)$, since this always gives the maximum possible number of sources. Amongst the proper approaches is traditional detection, whereby the smallest value of $\mu$ for which $L R\left(R_{\mu}\right)$ exceeds some threshold is taken to be the estimated number of sources $\hat{m}$.

In this study, we choose to employ the sphericity test [12],

$$
\begin{array}{ll}
H_{0}: \mathcal{E}\left\{C^{-\frac{1}{2}} \hat{R} C^{-\frac{1}{2}}\right\}=c_{0} I_{M} & \text { against } \\
H_{1}: \mathcal{E}\left\{C^{-\frac{1}{2}} \hat{R} C^{-\frac{1}{2}}\right\} \neq c_{0} I_{M}, & c_{0}>0
\end{array}
$$

whereby the definition of the LR for Gaussian mixtures becomes

$$
\gamma(C)=\left(\frac{\operatorname{det}\left(C^{-1} \hat{R}\right)}{\left[\frac{1}{M} \operatorname{tr}\left(C^{-1} \hat{R}\right)\right]^{M}}\right)^{N} \equiv \gamma_{0}^{N}(C) .
$$

Note that the $\operatorname{LR} \gamma(C)$ is just a normalized LF [5]. This means that from the ML point of view, ML optimization is equivalent to optimizing the $\operatorname{LR} \gamma(C)$ (13), and all its extrema are the same as for the LF. Unlike the LF, the $\operatorname{LR} \gamma(C)$ is normalized, i.e.,

$$
0 \leq \gamma(C) \leq 1
$$

and, most important, when $C=R$ (the true covariance matrix), the pdf of $\gamma(R)$ does not depend on $R$, i.e., is scenario free. Indeed

$$
\gamma_{0}(R)=\frac{\operatorname{det}\left[R^{-\frac{1}{2}} \hat{R} R^{-\frac{1}{2}}\right]}{\left(\frac{1}{M} \operatorname{tr}\left[R^{-\frac{1}{2}} \hat{R} R^{-\frac{1}{2}}\right]\right)^{M}}
$$

and according to [11]

$$
\hat{C}=R^{-\frac{1}{2}} \hat{R} R^{-\frac{1}{2}} \sim \mathcal{C W}\left(N, M, I_{M}\right)
$$

which is the complex Wishart distribution that is here completely specified by the sample volume $N$ and the array dimension $M$.

In [5], the well-known properties of $\gamma_{0}(R)$ for real-valued random Gaussian data [13] were derived for the complex (circular) Gaussian case. Instead of dealing with the rather cumbersome Meijer $G$ function derived in [5], we use direct Monte Carlo simulations to compute the pdf to the required precision. We then find threshold values $\alpha\left(P_{\mathrm{FA}}\right)$ for various probabilities of false alarm $P_{\mathrm{FA}}$ (i.e., incorrect identification, where the true
ML estimate is wrongly treated as containing an outlier) such that

$$
1-\int_{0}^{\alpha\left(P_{\mathrm{FA}}\right)} f\left(\gamma_{0}\right) d \gamma_{0}=P_{\mathrm{FA}} .
$$

Indeed, when $R \in \mathbb{R}_{\mu}$, i.e., $\mu \geq m$, then

$$
\max _{R_{\mu} \in \mathbb{R}_{\mu}} \gamma_{0}\left(R_{\mu}\right) \geq \gamma_{0}(R)
$$

and so for truly ML estimates, $P_{\mathrm{FA}}$ is the upper bound for the actual probability of false alarm. Therefore, the smallest value of $\mu$ for which

$$
\gamma_{0}\left(R_{\mu}\right)>\alpha\left(P_{\mathrm{FA}}\right)
$$

is taken to be the estimated number of sources $\hat{m}$.

Within this GLRT approach, $P_{\mathrm{FA}}$ may also be treated as the probability of overestimation; indeed, some number of sources $\mu>m$ could be found that has an LR exceeding the given $P_{\mathrm{FA}}$. This scenario-free thresholding does not require informationtheoretic criteria (ITC) [14] to be involved. Yet, the traditional uncertainty regarding $P_{\mathrm{FA}}$ (threshold-level) selection may be addressed by ITC, whereby

$$
\hat{m}=\arg \min _{\mu}\left[-\log L R\left(R_{\mu}\right)+\nu_{\mu}\right]
$$

where the ITC penalty term $\nu_{\mu}$ increases with the hypothesized number of sources $\mu$. If $d_{\mu}$ is the number of real-valued parameters that completely define the covariance matrix $R_{\mu}$, then typical choices are [14]

$$
\nu_{\mu}=\left\{\begin{array}{ll}
\nu_{\mathrm{AIC}} \equiv d_{\mu}, & \text { Akaike information criterion } \\
\nu_{\mathrm{MDL}} \equiv \frac{1}{2} d_{\mu} \log N, & \text { minimum description length } \\
\nu_{\mathrm{MAP}} \equiv \frac{5}{6} d_{\mu} \log N, & \text { maximum a posteriori } \\
& \text { probability }
\end{array} .\right.
$$

For our model of independent point Gaussian sources, $d_{\mu}=2 \mu$.

Interestingly, the famous Wax-Kailath ITC detection method [15] is based on the same sphericity test under the constraint that $C$ is a positive-definite (p.d.) Hermitian matrix whose $(M-\mu)$ smallest eigenvalues are equal.

Since the set of p.d. Hermitian matrices $\mathbb{H}_{\mu}$ whose smallest $(M-\mu)$ eigenvalues are equal includes the set of antenna arrayspecific covariance matrices $\mathbb{R}_{\mu}$, i.e., $\mathbb{R}_{\mu} \subset \mathbb{H}_{\mu}$, we must have

$$
\gamma_{0}\left(H_{\mu}^{\mathrm{opt}}\right) \geq \gamma_{0}\left(R_{\mu}^{\mathrm{ML}}\right) \geq \gamma_{0}(R) .
$$

Instead of the ITC criteria suggested by Wax and Kailath [15], we can again apply the "lower bound" thresholding (19)

$$
\gamma_{0}\left(H_{\mu}^{\mathrm{opt}}\right)=\frac{\prod_{j=\mu+1}^{M} \hat{\lambda}_{j}}{\left(\frac{1}{M-\mu} \sum_{j=\mu+1}^{M} \hat{\lambda}_{j}\right)^{M-\mu}}>\alpha\left(P_{\mathrm{FA}}\right) .
$$

Naturally, property (22) means that this may underestimate the number of sources. If this occurs, and the erroneous $\hat{m}$ is used for DOA estimation, the final test (18) will reject this model if 


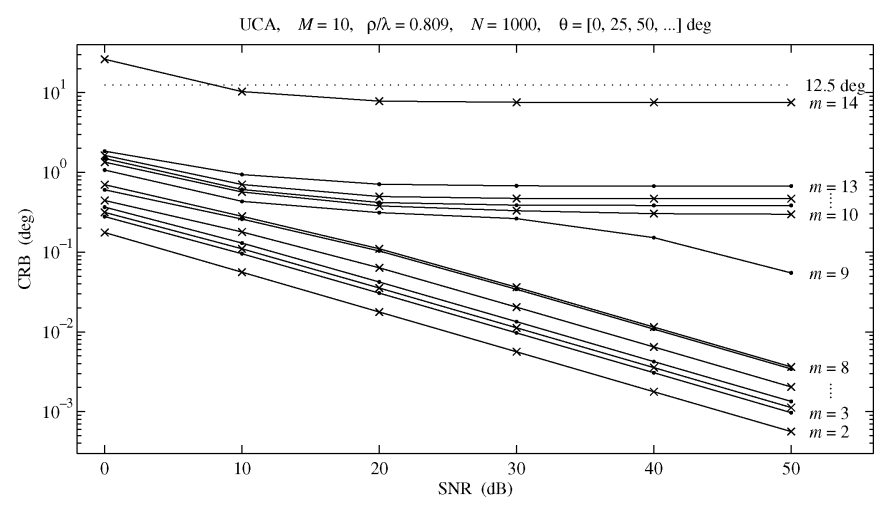

Fig. 1. Maximum Cramér-Rao bound for a ten-sensor uniform circular array and $m$ equiangular sources.

the underestimated model $R_{\hat{m}}$ does not have the expected match with the sample covariance matrix.

More often, we observe a different phenomenon: the Wax-Kailath test together with the originally proposed ITC, as well as with the thresholding (23), both give the true estimate for $m$ that could not be resolved even by ML estimation techniques [16]. This is the typical manifestation of "threshold conditions," where initially MUSIC and eventually even the ML technique generate a set of DOA estimates that may include completely erroneous DOA estimates for sources that are beyond practical (or "effective" [17]) resolution capabilities.

Whereas the Wax-Kailath Hermitian embedding $\mathbb{R}_{\mu} \subset \mathbb{H}_{\mu}$ applies for a "conventional" number of independent point sources $(m<M)$, only the general GLRT framework does not have such limitations; in fact, in [10], [18], and [19], we used it for detection-estimation for scenarios where $R$ does not have a "noise subspace." There, we considered a "superior" number of sources $(m \geq M)$ in sparse (nonuniform) minimum redundancy antenna arrays [10], [18], and also scenarios with spread (distributed) sources [19].

In this regard, it would be interesting to comment on the ability of a circular array to address a superior scenario. On one hand, an $M$-sensor UCA generates a Hermitian covariance matrix; while it is not Toeplitz, it does have a certain structure that was exploited in the rectification routine [8]. For a ten-sensor UCA, the covariance matrix $R$ is fully specified by 51 different real numbers, rather than the 91 numbers that would be required for an arbitrary ten-variate Hermitian matrix with constant diagonal. Clearly, the maximum number of identifiable sources is 25 in this case, notwithstanding the question whether or not this number could be identified in practice. On the other hand, it was noted in [20] that

...the effective number of sources that may be resolved by a circular antenna array at a fixed elevation is a function of the radius ... of the array and elevation angle. In particular, that number may be smaller than $M$, the number of sensors.

Obviously the issues associated with identifiability need to be better understood.

An insight into this problem is provided by a Cramér-Rao bound (CRB) analysis. Assuming known noise power, Fig. 1 shows the maximum CRB for increasingly large sets of equispaced DOAs in a ten-sensor UCA with $\rho / \lambda=$ $(1 / 4) \sin (\pi / M)=0.809$ as in [9], and 1000 snapshots. The CRB behavior up to eight sources follows the familiar pattern of increasing accuracy with increasing SNR. For ten or more sources, we see typical behavior for such superior scenarios, where the accuracy no longer depends on SNR beyond some threshold SNR (here, about $20 \mathrm{~dB}$ ) [21]. The $m=9$ case clearly shows a transition from "conventional" to "superior" nature. The CRBs for $10 \leq m \leq 13$ lie close to each other, and are significantly smaller than the intersource separation for SNRs above $10 \mathrm{~dB}$. For $m=14$, the potential accuracy drops dramatically; indeed, for all realistic SNRs, the CRB is comparable to the intersource separation. This makes it clear that the number of practically identifiable sources here cannot exceed $m=13$, which is significantly less than the number of sources suggested by this UCAs number of real degrees of freedom $\left(\mathrm{RDoF}=51\right.$, hence $\left.m_{\max }=25\right)$.

In fact, one successful attempt to resolve $m=M$ sources was reported in [9]. However, for the scenario considered in [9]

$$
\begin{aligned}
& M= 10, \quad N=100, \quad \frac{\rho}{\lambda}=\frac{1}{4} \sin \frac{\pi}{10}, \\
& \boldsymbol{\theta}_{10}= {[-160,-100,-90,-60,-30,} \\
&10,40,80,120,160]^{\circ}
\end{aligned}
$$

with a common SNR of $0 \mathrm{~dB}$, we found the CRB for the third (worst) source to be $12.8^{\circ}$, which is far too high to cope with the ten-degree separation between the second and third sources.

Unfortunately, even with sample support greater than the $N=1000$ in Fig. 1, "practically nonidentifiable" scenarios can frequently occur. Let us give an example that demonstrates this phenomenon. For the same ten-sensor UCA as above, consider the 14 sources

$$
\begin{gathered}
\boldsymbol{\theta}_{14}^{(1)}=[-175.8,-130.9,-117.7,-108.3,-89.2,-82.3 \\
8.1,85.4,106.1,135.3,136.9,141.8,164.5,172.7]^{\circ}
\end{gathered}
$$

with a common SNR of $0 \mathrm{~dB}$. Then, the different 14-source scenario

$$
\begin{aligned}
\boldsymbol{\theta}_{14}^{(2)}= & {[-176.5,-130.7,-116.4,-105.8,-85.6,-75.2} \\
& -22.4,8.1,47.9,85.4,106.1,136.7,146.7,168.3]^{\circ}
\end{aligned}
$$

with properly selected powers has the exact covariance matrix $R_{2}$ that is barely distinguishable from that of the first scenario according to the sphericity test

$$
\gamma_{0}\left(R_{1} \text { vs } R_{2}\right)=\frac{\operatorname{det}\left[R_{1}^{-1} R_{2}\right]}{\left(\frac{1}{M} \operatorname{tr}\left[R_{1}^{-1} R_{2}\right]\right)^{M}}=0.9994
$$

hence these two scenarios could not be reliably discriminated in practice.

This analysis shows that "superior" capabilities of UCAs are extremely limited. The compactness of the UCA and associated problems (unlike in sparse linear arrays) do not allow for the high angular resolution of a large number of sources. While the GLRT framework includes superior scenarios, and in fact, the Toeplitz embedding suggested in [8] (where a circular array covariance matrix is presented as a transformation of a virtual 
ULA's Toeplitz covariance matrix) could be used to initialize ML optimization, these possibilities are of academic interest only because of this "practical nonidentifiability."

Thus, practical nonidentifiability and ML performance breakdown are two sides of the same phenomenon, whereby severely erroneous solutions arise with extremely high LRs. The problem is that superior scenarios in UCA are quite likely to encounter practical nonidentifiability, which is due to the specific structure of the circular array [17]. Increasing the SNR and/or sample support cannot overcome this problem, as in the above example. On the contrary, ML performance breakdown for $m<M$ sources can be avoided by properly selecting SNR and/or sample support. For this reason, in what follows, we concentrate on performance improvement in the "threshold" region for conventional scenarios, using the proposed technique.

\section{Performance Breakdown of Subspace Methods: ARRAY GEOMETRY-FrEe GLRT PREDICTION AND CURE}

The first step of the traditional subspace-based detection-estimation approach is the Wax-Kailath technique [15] for estimating the number of sources; this tests the hypothesis regarding the equality of the smallest $(M-\mu)$ eigenvalues of $R_{\mu}$. The second step is to use a subspace technique such as MUSIC to compute the $\hat{m}$-variate set of DOA estimates. When the sample size $N$ and/or SNR are large enough to expect asymptotically efficient MUSIC-generated DOA estimates, then the performance of this approach will be high. In this "asymptotic domain," then, we expect the DOA and power estimates to yield by (13) very accurate LRs that are statistically indistinguishable from the LR of the true covariance matrix, hence any possible improvement in performance by use of the GLRT method would be marginal.

In fact, the current justification of this traditional two-step detection-estimation approach relies mainly on these asymptotic considerations. In practice, when the underlying scenario is unknown, this reliance does not allow us to verify how close the results are to the true ML condition. Note that a "perfect match" in terms of the LR (19) does not guarantee that detection-estimation results are free from outliers. For any given sample size and/or SNR, there should always exist a sufficiently small intersource separation such that the sources could not be discriminated by any technique. Underestimation of the number of sources in this case may or may not be treated as a severe error, considering the fact that the remaining (detected) sources are properly estimated.

Occurring more frequently is a different phenomenon that arises because Wax-Kailath detection and MUSIC estimation deal with different models, and so may have different thresholds (and in fact they do, see [16]). Indeed, testing the equality of the "noise-subspace" eigenvalues in an arbitrary Hermitian matrix may generate the correct number of sources that yet could not be properly estimated (resolved) even by the true ML technique. Typically in this case, a subset with a smaller number of estimated sources creates a model that is not "as likely" as the true set in terms of the LF/LR metric, while for the true number of sources that are beyond the ML resolution capability, we usually obtain a severely erroneous "noise-driven" DOA estimate.

This phenomenon is "ML performance breakdown," as distinct from "MUSIC performance breakdown." The latter deals with a set of DOA estimates that are below the LR bound in (19), while the former concerns a severely erroneous model that is "as likely" as the true one. In this regard, the GLRT approach (17)-(19) that finds the minimum number $\hat{m}$ of sources with DOA estimates that generate a covariance matrix model that statistically is as likely as the true covariance matrix can be used to improve the performance of the traditional Wax-Kailath detection + MUSIC estimation approach in the threshold area as follows.

\section{A. Wax-Kailath-MUSIC Performance Breakdown}

Manifestation: The set of MUSIC-generated DOA estimates for the detected number of sources $\hat{m}, \hat{\boldsymbol{\theta}}_{\hat{m}}$, gives the covariance matrix model $R\left(\hat{\boldsymbol{\theta}}_{\hat{m}}\right)$ that does not meet the threshold condition (19).

Reasons:

- $\hat{m}=m$ but MUSIC fails due to "subspace swap" [22]; or

- $\hat{m}<m$ for potentially well-resolvable sources (this is a significantly less probable event for the Wax-Kailath technique).

Goal: To correct the DOA estimates and the estimated number of sources, if necessary, in order to meet the GLRT condition (19).

\section{B. Overestimation}

Manifestation: The set of MUSIC-generated DOA estimates for the detected number of sources $\hat{m}, \hat{\boldsymbol{\theta}}_{\hat{m}}$, gives the covariance matrix model $R\left(\hat{\boldsymbol{\theta}}_{\hat{m}}\right)$ that does meet the threshold condition (19). The $(\hat{m}-1)$ subset of (modified) DOA estimates also meets the threshold condition (19).

Reason: $\hat{m} \geq m$ so that severely erroneous "noise-driven" DOA estimate(s) arise due to ML performance breakdown or overestimation.

Goal: To specify the minimum number of sources and their DOA estimates that meet the threshold condition (19).

\section{Algorithm}

Step 1: Our rectification scheme begins from a "quality assessment" of the DOA estimates $\hat{\boldsymbol{\theta}}_{\hat{m}}$ given by the Wax-KailathMUSIC method. We first estimate the white-noise power (if unknown) as

$$
\hat{p}_{0} \equiv \frac{1}{M-\hat{m}} \sum_{\hat{m}+1}^{M} \lambda_{j}
$$

and the vector of estimated source powers as [1]

$$
\begin{aligned}
\hat{\boldsymbol{p}}_{\hat{m}} \equiv \operatorname{diag}_{+}\{[ & \left.S^{H}\left(\hat{\boldsymbol{\theta}}_{\hat{m}}\right) S\left(\hat{\boldsymbol{\theta}}_{\hat{m}}\right)\right]^{-1} \\
& \left.\times\left(\hat{R}-\hat{\boldsymbol{p}}_{0} I_{M}\right)\left[S^{H}\left(\hat{\boldsymbol{\theta}}_{\hat{m}}\right) S\left(\hat{\boldsymbol{\theta}}_{\hat{m}}\right)\right]^{-1}\right\}
\end{aligned}
$$

where

$$
\operatorname{diag}_{+}\left\{x_{j}\right\} \equiv\left\{\begin{array}{ll}
x_{j}, & \text { for } x_{j} \geq 0 \\
0, & \text { for } x_{j}<0
\end{array} .\right.
$$

Other matching techniques that search for non-negative signal power estimates are also possible; in [10] and [18], for example, 
we used linear programming-based matching. Then, we form the MUSIC-specific model

$$
R_{\mathrm{MUSIC}} \equiv p_{0} I_{M}+S\left(\hat{\boldsymbol{\theta}}_{\hat{m}}\right) \hat{\boldsymbol{p}}_{\hat{m}} S^{H}\left(\hat{\boldsymbol{\theta}}_{\hat{m}}\right)
$$

and check the LR inequality (19).

If the threshold is met then we skip to Step 5, otherwise we continue.

Step 2: We perform a direct (unconstrained) gradient-type LR maximization over the set of $(2 \hat{m}+1)$ parameters $\left\{\hat{\boldsymbol{\theta}}, \hat{\boldsymbol{p}}, \hat{p}_{0}\right\}$ in the neighborhood of the MUSIC-generated estimates $\left\{\hat{\boldsymbol{\theta}}_{\hat{m}}, \hat{\boldsymbol{p}}_{\hat{m}}, \hat{p}_{0}\right\}$.

In rare cases, when the MUSIC estimate is in the convex proximity of an appropriate LR maximum, we expect to get a solution that exceeds the lower bound, and the same final check on overestimation as per Step 5 completes Step 2. Unfortunately, this desirable outcome is rare; since the LR optimization is erroneously initialized by MUSIC, the optimization usually converges to a local extremum that does not exceed the lower bound. Therefore, this set either still contains a MUSIC-generated outlier, or the number of sources has been underestimated.

Step 3: We now have to identify the specific reason for the negative outcome at Step 2 . We could conventionally test the underestimation hypothesis by applying Steps 1 and 2 for the $(\hat{m}+$ $j$ ) DOA estimates $(j=1,2, \ldots)$ generated by MUSIC from $(\hat{m}+j)$ sources. However, we propose a unified approach for this testing: we exclude in turn each source from the $\hat{m}$-variate model $R$ by defining

$$
\tilde{R}_{m j} \equiv R_{m}-\hat{p}_{j} S\left(\theta_{j}\right) S^{H}\left(\theta_{j}\right) \quad \text { for } \quad j=1, \ldots, m
$$

where $R_{m}$ is the covariance matrix model comprising the $m$ sources that are given by the LR-optimized MUSIC DOA estimates from Step 2, with their power estimates computed by (28) and (29).

We then compute the $m$ values $F_{j}(j=1, \ldots, m)$ of the $\mathrm{LR}$ $\gamma_{0}(C)$, as follows:

$$
F_{j} \equiv \gamma_{0}\left(\tilde{R}_{m j}\right) \equiv \frac{\operatorname{det}\left(\tilde{R}_{m j}^{-1} \hat{R}\right)}{\left[\frac{1}{M} \operatorname{tr}\left(\tilde{R}_{m j}^{-1} \hat{R}\right)\right]^{M}}
$$

and compare $\gamma_{0}\left(\tilde{R}_{m j}\right)$ with $\gamma_{0}\left(R_{m}\right)$. Any source whose excision from the model does not lead to a significant degradation in LR, i.e.,

$$
\gamma_{0}\left(\tilde{R}_{m j}\right) \approx \gamma_{0}\left(R_{m}\right)
$$

can be considered an outlier.

The rationale behind this approach is that an incorrect DOA estimate cannot contribute significantly to the LR, while the defect in LR is due to the fact that the correct DOA estimate is missing from $\tilde{R}_{m}$. (At this point, we are assuming the scenario is identifiable.) Hence, if excluded from the model, any erroneous source should not incur a significant additional degradation in LR, compared with the original LR for $\tilde{R}_{m}$. On the contrary, if a correctly estimated source is removed from the model, then a significant LR degradation will be observed.
Naturally, this simple procedure for determining outliers could be made more sophisticated; for example, a direct LR optimization could be used that is initialized by each $\tilde{R}_{m j}$, so that $F_{j}$ is then the true (but local) LR maximum in the neighborhood of each DOA set.

In what follows, the source with the maximum $F_{j}$ $\left(\approx \gamma_{0}\left(R_{m}\right)\right)$ is treated as the most probable outlier that must be replaced by a "proper" estimate. On the contrary, if for all $j$

$$
\gamma_{0}\left(\tilde{R}_{m j}\right) \ll \gamma_{0}\left(R_{m}\right)
$$

then underestimation of the number of sources has occurred, and only now may MUSIC be initiated for the augmented number of $(m+1)$ sources. In most cases, we observe a MUSIC outlier that does not significantly contribute to the LR (34) to be detected by (34).

In [5], which deals with uniform linear arrays (ULAs), we suggested an array geometry-specific (more computationally demanding) approach that is quite unlike the Wax-KailathMUSIC detection-estimation routine. This procedure was proposed for scenarios where MUSIC failed to meet the lowerbound condition (19). In essence, we first seek a p.d. Toeplitz covariance matrix estimate that meets the same threshold condition (19), and then gradually equalize the smallest $(M-j)$ $(j=M-2, M-3, \ldots)$ eigenvalues in this Toeplitz matrix, then define $\hat{m}$ as the smallest $j$ for which the "equalized" Toeplitz matrix exceeds the lower bound (19). This avoids "threshold" problems associated with the generic geometryindependent Wax-Kailath-MUSIC approach.

As we have seen, the UCA covariance matrix also has a particular structure, and in fact may be approximated by a Toeplitz matrix of a certain size [8]. Therefore, similar LR optimization routines to the ULA geometry-dependent case could also be proposed. Instead, we have suggested a geometry-free approach to "predict and cure" MUSIC outliers. In Section IV, we compare these two approaches for a ULA in order to justify this new approach.

Step 4: Having found the most likely outlier by removing each in turn, the next step is to replace the suspected outlier by a proper (ML) estimate. The GLRT philosophy prompted our following simple two-step rectification algorithm. Let $\tilde{R}^{(-1)}$ be the MUSIC-generated covariance matrix with one outlier removed from the model. We introduce the pseudo-spectrum-like function

$$
f^{(-1+1)}(\theta) \equiv \gamma_{0}\left(\tilde{R}^{(-1+1)}\right)
$$

where

$$
\tilde{R}^{(-1+1)} \equiv \tilde{R}^{(-1)}+\hat{p}(\theta) \boldsymbol{s}(\theta) \boldsymbol{s}^{H}(\theta)
$$

where $\hat{p}(\theta)$ is the estimate of the additional source power with azimuth $\theta$. We then simply find the maximum of the function $f^{(-1+1)}(\theta)$ and treat the DOA estimate

$$
\hat{\theta}^{(-1+1)} \equiv \arg \max _{\theta} f^{(-1+1)}(\theta)
$$


as the rectified outlier. In other words, to the $(m-1)$ reduced set of DOAs, we search all azimuths to find the most likely additional DOA. The next step is to use the new $m$ set of DOAs and powers to initialize a further application of the LR maximization routine.

If this final set of refined parameters $\left\{\hat{\boldsymbol{\theta}}, \hat{\boldsymbol{p}}, \hat{p}_{0}\right\}$ exceeds the LR lower bound, then it is accepted, otherwise it is assumed that the original set contained more than one outlier, and hence we repeat the outlier recognition and outlier rectification routines (Step 3) to eliminate further outliers. On rare occasions, this cycle may repeat indefinitely (so far, we have encountered it with probability less than $10^{-5}$ ), in which case the iterations are terminated at some maximum limit, and that set of parameter estimates is disregarded. Apart from true "false alarms," the cause of this rare occurrence is usually when MUSIC failed to initialize most of the DOA estimates, so other DOA estimation routines may be used for initialization (such as the DOA estimation bank method [23]). Even a random search may be used to find an acceptable solution (in the LR sense) that must always exist.

Recall that this outlier rectification routine is used only if the traditional detection-estimation process (Wax-Kailath then MUSIC, say), followed by LR (local) optimization, gives an unacceptable solution (i.e., with LR below the lower bound (19)).

Step 5: The previous four steps may give a model with optimally high LR, but one that still includes an outlier, due to the ML performance breakdown phenomenon (when the estimated number of sources is correct), or by occasional overestimation. Therefore, the procedure (33)-(34) must be finally applied to eliminate any possible extraneous estimates from the model so as to obtain the minimal number of estimated sources that meet the threshold condition.

Note that when the number of sources given by the WaxKailath technique must be reconsidered (in Steps 3 and 5), instead of the thresholding (19), we can still apply the "soft" ITC approach. However, since the appropriate thresholds need to be calculated anyway, we suggest that the accurate thresholding method for some $P_{\mathrm{FA}}$ is used.

One can see that the above outlier rectification routine is applicable to an arbitrary antenna array geometry.

\section{Performance Breakdown of Subspace Methods: SIMULATION RESULTS FOR UCAS AND ULAS}

To illustrate the efficiency of this outlier recognition and rectification method, we simulate a $M=10$-sensor UCA with $\rho / \lambda=(1 / 4) \sin (\pi / 10) \simeq 0.809$ so that the distance between two neighboring sensors is $\lambda / 2$. Consider the scenario with $m=$ 5 independent sources with 20-dB SNR per source, and $N=$ 300 snapshots. Four sources are uniformly distributed in azimuth, while the fifth DOA is placed at various close separations from the fourth, as follows:

$$
\boldsymbol{\theta}_{5}=\left[0^{\circ}, 30^{\circ}, 60^{\circ}, 90^{\circ}, \theta_{5}\right] ; \quad \theta_{5}=\left\{93^{\circ}, 91.6^{\circ}, 91^{\circ}\right\}
$$

These three separate DOA values for the fifth source $\theta_{5}$ have been specifically selected to demonstrate the transition from the MUSIC-specific performance-breakdown conditions, which can be efficiently rectified by our GLRT technique $\left(\theta_{5}=93^{\circ}\right)$,

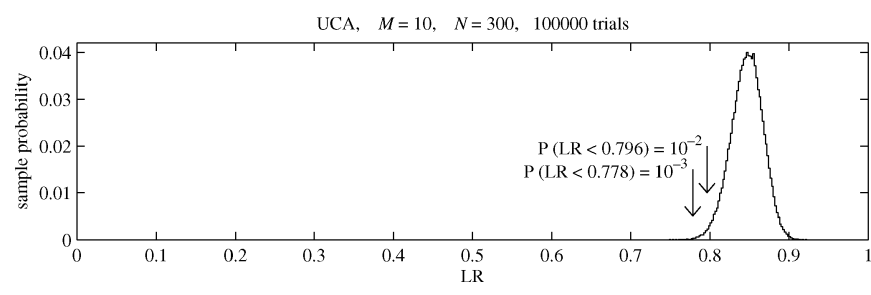

Fig. 2. Sample likelihood-ratio pdf for a ten-sensor UCA with 300 snapshots.

to the ML performance-breakdown conditions that could not be reliably rectified within the ML paradigm $\left(\theta_{5}=91^{\circ}\right)$.

The CRB for the fifth source is $0.36^{\circ}, 0.75^{\circ}$, and $1.46^{\circ}$ respectively. Thus, for $\theta_{5}=93^{\circ}$, the CRB predicts that the ultimate DOA estimation accuracy is sufficient for reliable separation of the fourth and fifth sources, since it is significantly smaller than the intersource separation of $3^{\circ}$. At the other extreme, the CRB exceeds the $1^{\circ}$ separation; thus, reliable separation of the last two sources at $90^{\circ}$ and $91^{\circ}$ is impossible. Still, all three locations of the fifth source are close enough to the fourth source to cause a significant number of MUSIC outliers.

In the course of Monte Carlo simulations, we are able to do something that we cannot in practical applications: since here we know the true covariance matrix $R$, as well as comparing the LR with the scenario-free threshold (19), we can also directly test

$$
\gamma_{0}\left(R_{\mu}\right) \geq \gamma_{0}(R)
$$

in every trial in order to assess the potential capabilities of proper ML DOA estimation that must always satisfy this inequality. We may also evaluate the degradations associated with the rectification routine proposed above. This "trick" is similar to the standard CRB calculation that also requires the true covariance matrix. Moreover, in dealing with the "preasymptotic-threshold" domain, ML performance assessment judged according to (40) is even more appropriate than the CRB analysis derived under asymptotic conditions.

Therefore, our outlier analysis is conducted first using the impractical "strict" condition (40) in order to assess additional degradation caused by practical thresholding (19), where probabilities of incorrect identification are set at $10^{-2}$ and $10^{-3}$. We computed the scenario-free pdf $f\left(\gamma_{0}\right)$ by direct Monte Carlo simulation with $10^{5}$ trials, which leads to the thresholds $P(L R<0.796)=10^{-2}$ and $P(L R<0.778)=10^{-3}$. This function (Fig. 2 ) is very well localized within the range $0.8 \lesssim L R \lesssim 0.9$.

Fig. 3 shows the sample pdf's for the least difficult scenario $\theta_{5}=93^{\circ}$, with the strict lower bound ("LR threshold type 0"). Table I summarizes detection-estimation results for this scenario. Fig. 3(a) shows the sample LR pdf for i) the (strict) lower bound $\gamma_{0}(R)$ (solid line), which is the same as in Fig. 2 but here is for 1000 Monte Carlo trials; ii) the MUSIC DOA estimates (given the true number of sources) $\gamma_{0}(\tilde{R})$ (dashed line); and iii) the LR-optimized MUSIC DOAs (Step 2, dotted line). We see that the vast majority of trials (in fact, $91.9 \%$ ) resulted in MUSIC LRs that were extremely poor compared with the strict lower bound (19). Application of our direct LR maximization 
TABLE I

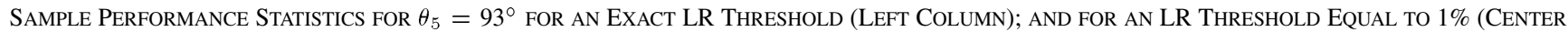
Column) and 0.1\% Probability of False Alarm (Right Column). The Number of NonRectified Outliers Is Zero in EACH CASE

\begin{tabular}{|c|r|r|r|}
\hline LR(MUSIC) < LR(threshold) & $91.9 \%$ & $84.2 \%$ & $83.1 \%$ \\
LR(optimization) $\geq$ LR(threshold) & $24.4 \%$ & $24.4 \%$ & $24.5 \%$ \\
LR(MUSIC) < LR(threshold) \& LR(optimization) $\geq$ LR(threshold) & $16.3 \%$ & $8.6 \%$ & $7.6 \%$ \\
\hline $\max$ CRB in $\boldsymbol{\theta}$ (degrees) & 0.36 & 0.36 & 0.36 \\
RMSE in $\hat{\boldsymbol{\theta}}$ after rectification and refinement (degrees) & 0.68 & 1.32 & 1.39 \\
\hline
\end{tabular}

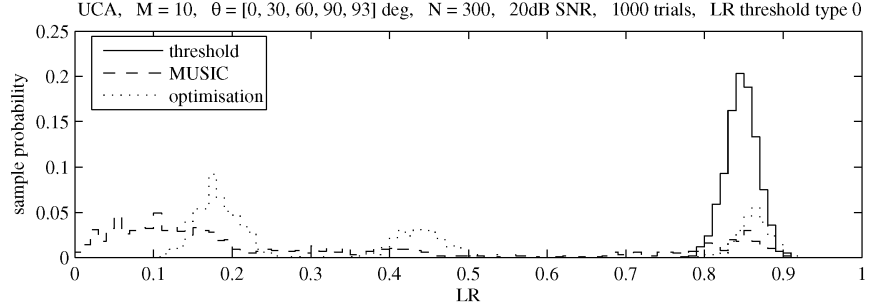

(a)

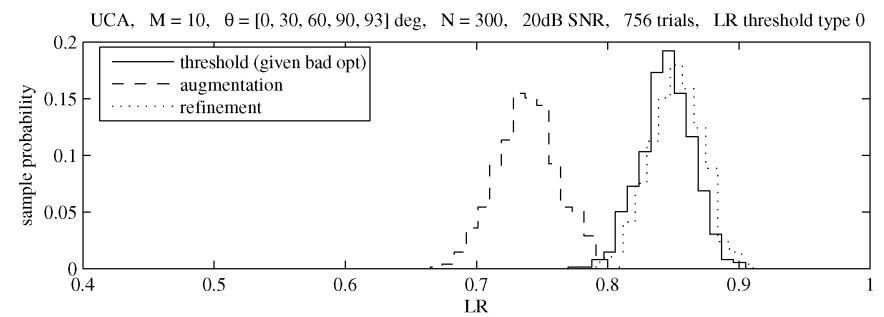

(b)

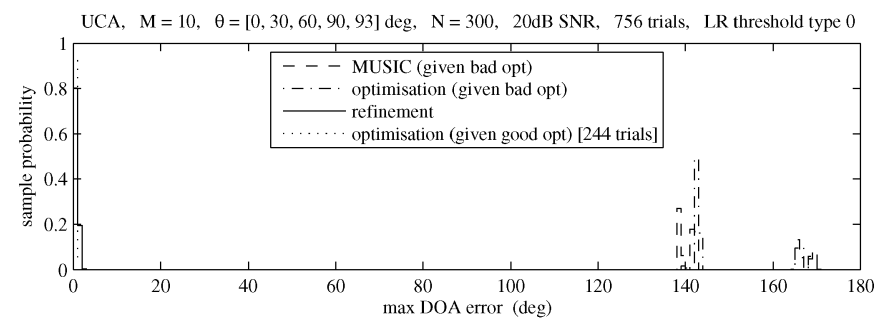

(c)

Fig. 3. Sample probability distributions of the maximum azimuth estimation error for $\theta_{5}=93^{\circ}$ and an exact LR threshold.

routine at Step 2, given this MUSIC initialization, improved the statistical LR only slightly, because the MUSIC outliers were usually far from the true DOAs. Indeed (see Table I), only $24.4 \%$ of trials resulted in a LR after optimization that exceeded the LR lower bound threshold. Since $8.1 \%$ of the original MUSIC trials exceeded the bound, optimization by itself was able to change ML-improper estimates to proper ones in only $16.3 \%$ of trials.

Fig. 3(b) illustrates the LR improvement during the outlier rectification process. For only those trials whose LR-optimization did not exceed the lower bound, we show the sample LR pdf for i) the (strict) lower bound $\gamma_{0}(R)$ (solid line); ii) the "outlier removed" (33) then "DOA augmented" (38) estimates (dashed line); and iii) the estimates after applying a further LR maximization (dotted line). We can see that even the first rectification step ii) shifts the LR dramatically to the right, quite close to the lower bound. Better still, the second rectification step iii) here pushes every single trial across the lower bound threshold.
Thus, in this case, the overall algorithm resulted in all 1000 trials having a LR "better than the true model."

The result of such LR improvements on DOA estimation accuracy are presented in Fig. 3(c), where we show the maximumDOA-error pdf for i) MUSIC estimates in those trials where application of the first LR maximization did not exceed the lower bound (dashed line); ii) subsequent LR maximization estimates (Step 2, dotted-dashed line); iii) subsequent outlier rectification (solid line); and iv) Step 2 LR maximization estimates in those trials where maximization did exceed the lower bound (dotted line).

First consider the iv) and ii) pdf's, whereby all 1000 Step 2 maximization trials (LR optimization in the neighborhood of the MUSIC DOAs) are respectively split into the group of those that were successful (244 trials) and those that were not (i.e., did not exceed the LR lower bound, 756 trials). Note that (see Table I) 81 of these 244 successful trials were already successful at the initial MUSIC estimate stage. The iv) pdf shows that there was not a single outlier present in the 244 trials, meaning that these trials could be considered "MUSIC-successful," despite the fact that 163 were pushed across the "strict" LR threshold by Step 2 maximization. On the contrary, the ii) pdf shows that each of the 756 optimization-unsuccessful trials contained outlier(s). The iii) pdf shows that all 756 trials were then successfully rectified, not only in terms of LR, but by eliminating all DOA estimation outliers. However, the final RMSE obtained for DOA estimation of $0.68^{\circ}$ is significantly above the CRB of $0.36^{\circ}$. More specifically, this error is obtained for the worst DOA $\theta=90^{\circ}$, with the sample bias $0.1735^{\circ}$ and standard deviation 0.66 , which is still high above the CRB. In this and the following tables, we report the root mean-square error (RMSE) only, since everywhere the bias is comparatively small and the standard deviations only marginally smaller than the RMSE. The only reasonable explanation is that, within the "threshold region," the likelihood function is not accurately approximated by its usual second-order expansion within the neighborhood of the true parameters.

The above scenario demonstrates the properties of an example where there is a significant distinction between MUSIC and MLoptimal performance; the latter is achievable only if an LR maximization algorithm with guaranteed global extremum exists, that of course must meet the "strict" threshold inequality (40).

As mentioned, we have presented results involving the strict (but impractical) lower bound (40) to investigate our technique's ultimate performance. We repeated the above experiment using practical thresholding (19), where the probabilities of false alarm are set at both $10^{-2}$ (hence $\alpha=0.796$ ) and $10^{-3}(\alpha=0.778)$. The results appear in the remainder of Table I. In both cases, almost the same number of trials 
TABLE II

SAME AS TABLE I BUT FOR $\theta_{5}=91.6^{\circ}$

\begin{tabular}{|c|r|r|r|}
\hline LR(MUSIC) < LR(threshold) & $100 \%$ & $99.5 \%$ & $98.4 \%$ \\
LR(optimization) $\geq$ LR(threshold) & $0.2 \%$ & $1.7 \%$ & $6.9 \%$ \\
LR(MUSIC) < LR(threshold) \& LR(optimization) $\geq$ LR(threshold) & $0.2 \%$ & $1.2 \%$ & $5.3 \%$ \\
\hline max CRB in $\boldsymbol{\theta}$ (degrees) & 0.75 & 0.75 & 0.75 \\
RMSE in $\hat{\boldsymbol{\theta}}$ after rectification and refinement (degrees) & 1.82 & 1.99 & 2.01 \\
number of nonrectified outliers & 0 & 17 & 69 \\
\hline
\end{tabular}

TABLE III

SAME AS TABLE I BUT FOR $\theta_{5}=91^{\circ}$

\begin{tabular}{|c|r|r|r|}
\hline LR(MUSIC) < LR(threshold) & $98.6 \%$ & $70.8 \%$ & $56.3 \%$ \\
LR(optimization) $\geq$ LR(threshold) & $6.9 \%$ & $51.2 \%$ & $68.9 \%$ \\
LR(MUSIC) < LR(threshold) \& LR(optimization) $\geq$ LR(threshold) & $5.5 \%$ & $22.0 \%$ & $25.2 \%$ \\
\hline max CRB in $\boldsymbol{\theta}$ (degrees) & 1.46 & 1.46 & 1.46 \\
RMSE in $\hat{\boldsymbol{\theta}}$ after rectification and refinement (degrees) & 2.94 & 3.35 & 4.20 \\
number of nonrectified outliers & 68 & 503 & 683 \\
number of eliminated sources (Step 5) & 0 & 2 & 11 \\
\hline
\end{tabular}

exceeded the bound after Step 2 optimization (244 or 245). These trials are the same trials that passed the threshold for the previous exact bound, having been rectified by the first LR maximization. This is another demonstration of the observation that $16.3 \%$ of trials did not contain a MUSIC outlier and were only marginally below the exact bound. In both cases all trials that failed the threshold after Step 2 optimization have been rectified. While none of the MUSIC-induced outliers survived, practical thresholding gave a final DOA estimation accuracy that was noticeably inferior to strict thresholding (see Table I). This degradation can be attributed to the fact that some trial estimates, whilst being above the bound, are still somewhat removed from the true LR maximum.

Since the rectified LR in all cases is beyond the lower bound, with all trials being properly identified, detection-estimation performance for this scenario is close to the ultimate. In fact, the Wax-Kailath MAP and MDL detectors achieve $100 \%$ correct detection, hence all of our 1000 trials have been properly identified (i.e., no outliers).

Finally, in this case, there were no sources eliminated by Step 5 as ones that do not contribute to the LR.

To summarize, all 1000 trials have been properly addressed by our GLRT rectification scheme, compared with only 244 by MUSIC.

The second scenario with $\theta_{5}=91.6^{\circ}$ was chosen to demonstrate the transition from MUSIC to ML performance breakdown. Here the maximum $\mathrm{CRB}\left(0.75^{\circ}\right)$ is approximately half the smallest intersource separation. In a similar format as above, Table II summarizes the estimation results for this more difficult scenario. While all of our 1000 trials initially failed to exceed the exact bound, the MUSIC estimates before and after optimization are statistically much closer to the LR bound than in the previous $\theta_{5}=93^{\circ}$ case. For the strict bound, two trials gave optimized LRs that were higher than $L R(R)$, and neither of these were outliers; all true outliers were later rectified. For the two practical bounds, on the other hand, all 17 and 69 such trials contained outliers. This shows the difference between this and the previous example. In this case, the Wax-Kailath MAP and MDL detectors also achieve 100\% correct detection; however, practical identification rate is not ideal, as per the previous case $\theta_{5}=93^{\circ}$.

This example illustrates the transition from ideal ML conditions (where we always have $L R\left(R_{\mu}\right)>L R(R)$ ) to the regime where our rectification comes to the fore. While not a single outlier "survived" rectification with the strict lower bound, 17 (respectively 69) trials exceeded the threshold calculated for $P_{\mathrm{FA}}=10^{-2}$ (respectively, $10^{-3}$ ). These outliers were directly produced by the optimization in Step 2 in the vicinity of the MUSIC estimates. Interestingly, none of these outliers were excluded in Step 5. In each case, the outlier was properly identified by Step 5 as least contributing to the LR, but LR optimization in the vicinity of the remaining (correct) four DOA estimates was unable to exceed the threshold. This once again indicates ML performance breakdown that stems from the fact that threshold conditions for detection are significantly different from those for estimation [16]. Indeed, the set of correct estimates for only four sources cannot be "as likely" as the true five-source scenario, while proper resolution of the given five-source scenario is unattainable.

Note, however, that here we have only a $1.7 \%$ (respectively, $6.9 \%$ ) probability of rectification breakdown, whereas MUSIC failed in $99.9 \%$ of trials.

For our third and most difficult scenario with $\theta_{5}=91^{\circ}$ (Table III), the ML performance breakdown is more profound. Almost all trials that passed the strict lower bound still contain outliers (68 out of 69). Moreover, even estimates that are close enough to the true ones to have a very high LR still have an RMSE that exceeds the intersource separation. Hence, this is an example of scenarios that are beyond the ML threshold conditions, i.e., could not be resolved by any ML means, despite the fact that for this scenario the Wax-Kailath MAP and MDL detectors achieve $86.4 \%$ and $99.8 \%$ correct detection respectively. This probability of correct detection is again outstandingly higher than the probability of correct 
TABLE IV

SAmple SiX-Source Performance Statistics For the Three TyPes of LR ThresholdS

\begin{tabular}{|c|c|c|c|}
\hline UCA, $M=10, N=300,20 \mathrm{~dB}$ SNR, 1000 trials, $\theta=[-3,0,30,60,90,93]^{\circ}$ & LR exact & $10^{-2} P_{F A}$ & $10^{-3} P_{F A}$ \\
\hline LR(MUSIC) $<$ LR(threshold) & $99.8 \%$ & $99.7 \%$ & $99.7 \%$ \\
\hline LR(opt) $\geq \operatorname{LR}$ (threshold) & $0.8 \%$ & $1.0 \%$ & $0.7 \%$ \\
\hline LR(MUSIC) $<$ LR(threshold) \& LR(opt) $\geq$ LR(threshold) & $0.6 \%$ & $0.7 \%$ & $0.4 \%$ \\
\hline prob. outlier identification was correct, given $\operatorname{LR}(\mathrm{opt})<\mathrm{LR}$ (threshold) & $44.1 \%$ & $44.0 \%$ & $44.2 \%$ \\
\hline number of iterations required to reach LR threshold & $\left|\begin{array}{llll}0 & 1 & 2 & 3\end{array}\right|$ & $\begin{array}{llll}0 & 1 & 2 & 3\end{array}$ & $\begin{array}{llll}0 & 1 & 2 & 3\end{array}$ \\
\hline number of trials & 81947962 & 101927971 & 71967952 \\
\hline $\max \mathrm{CRB}$ in $\theta$ (degrees) & 0.61 & 0.61 & 0.61 \\
\hline max total error in $\theta_{r e f}$, given LR(opt) $<\mathrm{LR}$ (threshold) (degrees) & 1.23 & 1.76 & 1.82 \\
\hline
\end{tabular}

detection by MUSIC $(0 \%)$. The complete failure of MUSIC is not surprising, given that the CRB here exceeds the intersource separation. Less predictable is that only 68 trials had the LR for the severely erroneous set exceeding $L R(R)$, and in none of these 68 trials did the LR for the optimized four-source scenario exceed this bound at Step 5. This example clearly demonstrates the inherent discrepancy between detection and estimation threshold conditions for the "ideal" GLRT method.

For "practical" thresholding, the number of outliers that survived comparison is significantly greater (503 and 683, respectively), with an almost negligible number of trials ( 2 and 11 respectively) being rectified by Step 5 as a four-source scenario with an outlier properly deleted from the DOA set. Even for this extremely difficult case, $32 \%-50 \%$ of trials were rectified by our "practical" scheme, though naturally the accuracy achieved over the rectified ensemble is significantly worse than the CRB.

This family of three examples has demonstrated the ability of our GLRT technique to "fill the gap" between the MUSIC- and the intrinsic ML-threshold conditions in UCAs.

We next briefly investigate our algorithm's capacity to rectify multiple outliers by considering the six-source scenario

$$
\boldsymbol{\theta}_{6}=\left[-3^{\circ}, 0^{\circ}, 30^{\circ}, 60^{\circ}, 90^{\circ}, 93^{\circ}\right]
$$

with the same $N=300$ and 20-dB SNR. Table IV shows similar data to before, but also the number of iterations required to reach the respective LR threshold (recall that outliers are rectified one at a time). We see that two iterations were almost always sufficient, as expected.

Finally, despite the efficient performance demonstrated above by our geometry-free GLRT rectification, an important question remains regarding the comparison between this and some geometry-specific LR optimization method. For this purpose, we now compare the efficiency of our new technique with the one we introduced in [5] for ULAs, where we considered the scenario

$$
\begin{aligned}
M & =5, \quad \frac{d}{\lambda}=0.5, \quad N=100, \quad m=3, \\
\sin \boldsymbol{\theta} & =[-0.40,0,0.06]
\end{aligned}
$$

with a common SNR of $20 \mathrm{~dB}$. For this scenario with $\mathrm{CRB}_{\min }=$ 0.010 , we reported that MUSIC failed in 541 out of 1000 Monte
Carlo trials and produced outliers with extremely low LR; in the remaining 459 trials, MUSIC produced acceptable results.

As mentioned above, in order to rectify these poor MUSIC results, we ignored them and launched a direct LR optimization that was based on Toeplitz covariance matrix properties. We used the Gohberg-Semencul routine of [24] to initialize the optimization over the set of p.d. Toeplitz matrices. In that study, we investigated the potential capabilities of that approach by continuing to search for an "appropriate" Toeplitz matrix until we achieved $L R(T) \geq L R(R)$, i.e., the "strict" (impractical) lower bound. This specific "Toeplitz optimization" routine did not give a $100 \%$ success rate; more precisely, in 45 trials the optimization did not reach the strict lower bound. Of these, the optimization routine converged to inappropriately low LR values in only seven trials, and in fact all these seven trials contained an outlier. The remaining 57 trials had relatively high LR, and in only 34 out of 64 cases did the LR exceed that of the true covariance matrix. Hence, the overall improvement achieved by our [5] technique was the reduction of 541 MUSIC outliers to 64.

Table V introduces the results of our scenario-free rectification scheme on the same example (42). For the same set of data, MUSIC produces 538 outlier trials. As in [5], here we define an outlier as a DOA estimate with an error that exceeds half of the smallest separation between sources $\left(\delta=0.03 \simeq 1.71^{\circ}\right)$. We see that Step 2 rectifies 370 outliers, while Step 4 rectifies only 80 outliers. This means that our current GLRT routine (with the strict lower bound) failed to detect ML performance breakdown in approximately the same number of trials ( 80 versus 64$)$. Whereas all our 80 "new" outliers are indeed "more likely" than the true parameters, compared with 34 instances in [5], the overall performance here is practically the same. Computationally though, the geometry-specific algorithm is much more involved.

In [5], we did not investigate a "practical" geometry-specific algorithm that is based on the appropriate selection of a Toeplitz covariance matrix from statistical thresholds. This analysis has now been performed for the new geometry-free rectification method, with the results shown in Table V. These results look different from our UCA results mainly because of the much more stringent outlier condition. If, for example, we define an outlier as an estimate with an error of less than $5^{\circ}$, then MUSIC fails in 526 cases, which means that most of the 538 MUSIC outliers in Table V really are severely erroneous. The number of $5^{\circ}$-outliers not rectified by Step 2 optimization is now between 
TABLE V

SAMPLE PERFORMANCE STATISTICS FOR THE ULA EXAMPLE

\begin{tabular}{|l|r|r|r|}
\hline ULA, $M=5, N=100,20 \mathrm{~dB}$ SNR, 1000 trials, $\sin \theta=[-0.40,0,0.06]$ & LR exact & $10^{-2} P_{F A}$ & $10^{-3} P_{F A}$ \\
\hline LR(MUSIC) < LR(threshold) & $77.8 \%$ & $58.6 \%$ & $56.2 \%$ \\
LR(opt) $\geq$ LR(threshold) & $87.1 \%$ & $90.3 \%$ & $91.0 \%$ \\
LR(MUSIC) < LR(threshold) \& LR(opt) $\geq$ LR(threshold) & $64.9 \%$ & $48.9 \%$ & $47.2 \%$ \\
LR(opt) < LR(threshold) \& LR(rect) < LR(threshold) & $0.3 \%$ & $0 \%$ & $0 \%$ \\
number of MUSIC outliers $\left(\delta<1.71^{\circ}\right.$ ) & 538 & 538 & 538 \\
number of outliers not rectified by Step 2 opt & 168 & 345 & 348 \\
number of outliers not rectified by Step 4 rect & 80 & 265 & 270 \\
number of eliminated sources & 0 & 0 & 0 \\
\hline max CRB in $\theta$ (degrees) & 0.55 & 0.55 & 0.55 \\
RMSE in $\hat{\theta}$ after rectification and refinement (degrees) & 0.88 & 1.26 & 1.13 \\
\hline
\end{tabular}

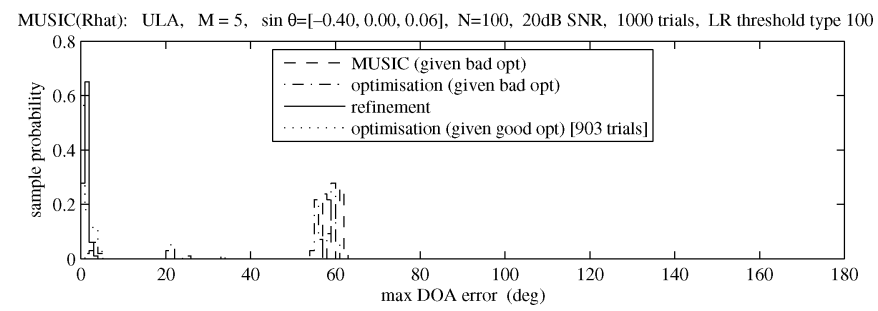

Fig. 4. Histogram of maximum errors in the DOA estimate at various stages in the ULA example.

only 85 and 93 . None of the $5^{\circ}$-outliers survived our rectification routine with the strict lower bound, while only one and 15 trials survived for the practical bounds with $P_{\mathrm{FA}}$ of $10^{-2}$ and $10^{-3}$, respectively.

Fig. 4 shows the sample pdf of the (worst) DOA estimation error at different stages of our rectification process for $P_{\mathrm{FA}}=$ $10^{-2}$. The severe MUSIC outliers $\left(\sim 60^{\circ}\right)$ have been mostly eliminated by the Step 2 optimization (910 trials). Those trials where Step 2 failed to reach the threshold ("given bad opt") are completely rectified by the refinement step. The tail of the error pdf vanishes at around $6^{\circ}$, but all severe MUSIC outliers are successfully rectified.

In summary, our numerical experiments make it clear that the GLRT technique, supported by the LR lower bound, gives a significant performance improvement over MUSIC in the "threshold region" for both UCAs and ULAs.

\section{SUMMARY AND CONCLUSION}

In this paper, we have modified our GLRT detection-estimation scheme to treat independent Gaussian sources for scenarios that could not be successfully addressed by conventional high-resolution techniques. We considered scenarios that lie in the problematic "threshold region" where subspace techniques (such as MUSIC) experience a sudden and dramatic "performance breakdown."

Our approach involves LR maximization that is considered successful if the result gives a LR that exceeds (statistically) that of the true model. We exploited the invariance property of the introduced LR which is the scenario-free nature of its pdf with respect to the true covariance matrix. Based on this property, we were able to precalculate thresholds that the optimized LR is compared against for any desired probability of false alarm (where a truly ML solution is wrongly determined to be an outlier). This pdf and the corresponding thresholds are exact and do not involve asymptotic considerations that are inappropriate in the threshold region. In simulation studies, we have been able to also use a "strict" comparison of the optimized LR with the one calculated from the true covariance matrix; this allows us to investigate the ultimate performance of our scheme and evaluate additional degradations caused by the statistical thresholding.

Comparing the LR of any set of estimates with a scenario-free threshold is a simple way to find non-ML estimates, including (say) MUSIC-specific outliers with an inappropriately low LR. We proposed a method of "rectifying" these inappropriate estimates that involves finding and replacing outliers by "proper" estimates, where the LR threshold is exceeded. This method is based on the straightforward observation that a set with low LR should have at least one missing correct estimate, whereas an outlier should have minimal contribution to the LR compared with the remaining proper estimates in the set.

Our method is applicable to any antenna array geometry, and has been tested for both ULAs and UCAs. Indeed, we compared the results against a different (geometry-specific) algorithm of ours [5] for ULAs. This showed that the new geometry-free method is practically as efficient as the earlier ULA-specific Toeplitz matrix approach.

We demonstrated that the efficiency of our GLRT-based rectification scheme, and in fact, of any ML-based scheme is limited by so-called "ML performance breakdown," which occurs when outliers give LRs that exceed that of the true model. The well-known intrinsic difference between detection and resolution thresholds could not be completely overcome by any approach, unlike the "gap" between MUSIC-specific and ML performance breakdown conditions. We presented scenarios where a MUSIC failure rate of $75 \%$ was completely rectified, and others where a 99\% MUSIC failure rate was rectified to a $1.7 \%-7 \%$ ML-breakdown failure rate.

While our GLRT method can be applied to detect-estimate more independent sources than sensors, we showed that UCA have a very limited capability for such "superior" scenarios (unlike minimum redundancy linear arrays). 


\section{REFERENCES}

[1] B. Ottersten, M. Viberg, P. Stoica, and A. Nehorai, "Exact and large sample maximum likelihood techniques for parameter estimation and detection in array processing," in Radar Array Processing, ser. Springer Series in Information Sciences, S. Haykin, J. Litva, and T. Shepherd, Eds. Berlin, Germany: Springer-Verlag, 1993, vol. 25, ch. 4, pp. 99-151.

[2] P. Stoica and K. Sharman, "Maximum likelihood methods for direction of arrival estimation," IEEE Trans. Acoust. Speech, Signal Process., vol. 38, no. 7, pp. 1132-1143, Jul. 1990.

[3] P. Stoica and A. Nehorai, "Performance study of conditional and unconditional direction-of-arrival estimation," IEEE Trans. Acoust. Speech, Signal Process., vol. 38, no. 10, pp. 1783-1795, Oct. 1990.

[4] Y. Abramovich and N. Spencer, "DOA estimation performance breakdown: a new approach to prediction and cure," in Proc. EUSIPCO, Toulouse, France, 2002, vol. 1, pp. 89-92.

[5] Y. Abramovich, N. Spencer, and A. Gorokhov, "Bounds on maximum likelihood ratio-Part I: Application to antenna array detection-estimation with perfect wavefront coherence," IEEE Trans. Signal Process., vol. 52, no. 6, pp. 1524-1536, Jun. 2004.

[6] D. Davies, "Circular arrays," in The Handbook of Antenna Design, A. Rudge, Ed. Stevenage, U.K.: Peregrinus, 1987, vol. 2, ch. 12.

[7] H. van Trees, Optimum Array Processing. New York: Wiley, 2002, vol. 4, Detection, estimation, and modulation theory.

[8] P. Forster, "Generalized rectification of cross spectral matrices for arrays of arbitrary geometry," IEEE Trans. Signal Process., vol. 49, no. 5, pp. 972-978, May 2001.

[9] J.-J. Fuchs, "On the application of the global matched filter to DOA estimation with uniform circular arrays," IEEE Trans. Signal Process., vol. 49, no. 4, pp. 702-709, Apr. 2001.

[10] Y. Abramovich, N. Spencer, and A. Gorokhov, "Detection-estimation of more uncorrelated Gaussian sources than sensors in nonuniform linear antenna arrays-Part I: Fully augmentable arrays," IEEE Trans. Signal Process., vol. 49, no. 5, pp. 959-971, May 2001.

[11] M. Siotani, T. Hayakawa, and Y. Fujikoshi, Modern Multivariate Statistical Analysis. Columbus, OH: Amer. Scientific Press, 1985.

[12] R. Muirhead, Aspects of Multivariate Statistical Theory. New York: Wiley, 1982.

[13] P. Consul, "The exact distributions of likelihood criteria of different hypotheses," in Multivariate Analysis II, P. Krishnaian, Ed. New York: Academic, 1969, pp. 171-181.

[14] P. Djurić, "A model selection rule for sinusoids in white Gaussian noise,” IEEE Trans. Signal Process., vol. 44, no. 7, pp. 1744-1757, Jul. 1996.

[15] M. Wax and T. Kailath, "Detection of signals by information theoretic criteria," IEEE Trans. Acoust., Speech, Signal Process., vol. 33, no. 2, pp. 387-392, Apr. 1985.

[16] H. Lee and F. Li, "Quantification of the difference between detection and resolution thresholds for multiple closely spaced emitters," IEEE Trans. Signal Process., vol. 41, no. 6, pp. 2274-2277, Jun. 1993.

[17] M. Doron and E. Doron, "Wavefield modeling and array processing," IEEE Trans. Signal Process., vol. 42, no. 10, pp. 2549-2580, Oct. 1994.

[18] Y. Abramovich, N. Spencer, and A. Gorokhov, "Detection-estimation of more uncorrelated Gaussian sources than sensors in nonuniform linear antenna arrays-Part II: Partially augmentable arrays," IEEE Trans. Signal Process., vol. 51, no. 6, pp. 1492-1507, Jun. 2003.

[19] — - "Bounds on maximum likelihood ratio-Part II: Application to antenna array detection-estimation with imperfect wavefront coherence," IEEE Trans. Signal Process., vol. 53, no. 6, pp. 2046-2058, Jun. 2005.

[20] A. Tewfik and W. Hong, "On the application of uniform linear array bearing estimation techniques to uniform circular arrays," IEEE Trans. Signal Process., vol. 40, no. 4, pp. 1008-1011, Apr. 1992.

[21] Y. Abramovich, D. Gray, A. Gorokhov, and N. Spencer, "Positive-definite Toeplitz completion in DOA estimation for nonuniform linear antenna arrays-Part I: Fully augmentable arrays," IEEE Trans. Signal Process., vol. 46, no. 9, pp. 2458-2471, Sep. 1998.

[22] M. Hawkes, A. Nehorai, and P. Stoica, "Performance breakdown of subspace-based methods: prediction and cure," in Proc. Int. Conf. Acoustics, Speech, Signal Processing (ICASSP), Salt Lake City, UT, 2001, vol. 6, pp. 405-408.
[23] A. Gershman, "Pseudo-randomly generated estimator banks: A new tool for improving the threshold performance of direction finding," IEEE Trans. Signal Process., vol. 46, no. 5, pp. 1351-1364, May 1998.

[24] I. Gohberg and A. Semencul, "On the inversion of finite Toeplitz matrices and their continuous analogues," (in Russian) Mat. Issled., vol. 7, no. 2, pp. 201-223, 1972.

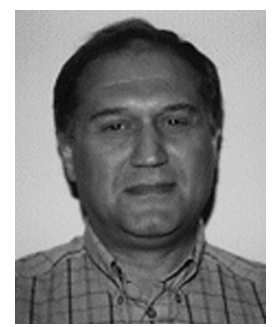

Yuri I. Abramovich (M'96-SM'06) received the Dipl.Eng. (Hons.) degree in radio electronics and the Cand.Sci. degree (Ph.D. equivalent) in theoretical radio techniques both from the Odessa Polytechnic University, Odessa (Ukraine), Russia, in 1967 and 1971, respectively, and the D.Sc. degree in radar and navigation from the Leningrad Institute for Avionics, Leningrad, Russia, in 1981

From 1968 to 1994, he was with the Odessa State Polytechnic University, Odessa, Ukraine, as a Research Fellow, Professor, and ultimately as Vice-Chancellor of Science and Research. Since 1994 to 2006, he was at the Cooperative Research Centre for Sensor Signal and Information Processing (CSSIP), Adelaide, Australia. Since 2000, he has been with the Australian Defence Science and Technology Organisation (DSTO), Adelaide, Australia, as Principal Research Scientist, seconded to CSSIP until its closure. His research interests are in signal processing (particularly spatio-temporal adaptive processing, beamforming, signal detection, and estimation), its application to radar (particularly over-the-horizon radar), electronic warfare, and communication.

Dr. Abramovich served as Associate Editor of the IEEE TRANSACTIONS ON SIGNAL PROCESSING from 2002 to 2005.

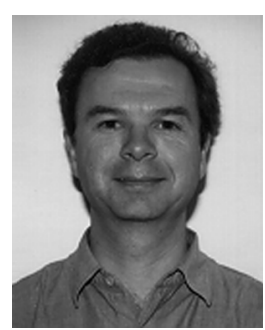

Nicholas K. Spencer received the B.Sc. (Hons.) degree in applied mathematics and the M.Sc. degree in computational mathematics from the Australian National University, Canberra, in 1985 and 1992, respectively.

He has been with the Australian Department of Defence, Canberra; the Flinders University of South Australia, Adelaide; the University of Adelaide; and the Australian Centre for Remote Sensing, Canberra; and the Cooperative Research Centre for Sensor Signal and Information Processing (CSSIP), Adelaide, Australia, in the areas of computational and mathematical sciences. He is currently a Senior Researcher at Adelaide Research \& Innovation Pty, Ltd. (ARI), Australia. His research interests include array signal processing, parallel and supercomputing, software best practice, human-machine interfaces, multilevel numerical methods, modeling and simulation of physical systems, theoretical astrophysics, and cellular automata.

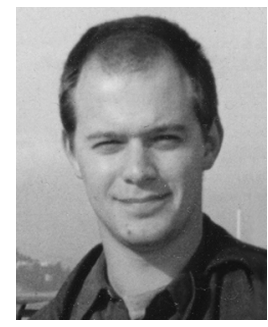

Alexei Y. Gorokhov (M'96) received the Ms.D. degree from the Odessa Polytechnic University (OPU), Odessa, Ukraine, in 1993 and the Ph.D. degree from the École Nationale Supérieure des Télécommunications, Paris, France, in 1997

From 1993 to 1994, he served as a Research Engineer with the research laboratory of the OPU. Since October 1997, he has been affiliated with the Centre National de la Recherche Scientifique (CNRS), Paris, France, where he holds a research position. From January 2000 to January 2004, he served as a Senior Scientist within the DSP group of Philips Research Laboratories, Eindhoven, The Netherlands, on long-term leave from CNRS. Since January 2004, he has been with Qualcomm, Inc., San Diego, CA. His general interests cover wireless communications, spectral analysis, statistical signal processing, and information theory. His current research is mainly focused on error control coding, multiuser equalization, and antenna diversity for wireless communications. 